\title{
Does Obesity-Related Hemodilution of Carcinoembryonic Antigen Exist in Non-Small Cell Lung Cancer Patients?
}

\author{
Masaki Tomita $^{\mathrm{a}, \mathrm{c}}$, Takanori Ayabe ${ }^{\mathrm{a}}$, Kunihide Nakamura ${ }^{\mathrm{b}}$
}

\begin{abstract}
Background: Previous investigations reported inverse relationship between prostate-specific antigen concentration and body mass index (BMI). These results have been explained by a hemodilution effect among obese men. However, the hemodilution of serum carcinoembryonic antigen (CEA) concentration in obese patients with non-small cell lung cancer (NSCLC) has not been ever reported.
\end{abstract}

Methods: Consecutive 381 NSCLC patients were enrolled. A body surface area (BSA)-based and a hematocrit (HCT)-based equations were applied for plasma volume (PV) estimation. The relationship between $\mathrm{BMI}$ and $\mathrm{PV}$, serum CEA concentration and CEA amount, representing the total amount of CEA protein within the circulation, were examined.

Results: Higher BMI was significantly associated with higher PV (P $<0.001)$. However, serum CEA concentration was not significantly associated with increasing BMI. Furthermore, there was no significant association between BMI and CEA amount. The 5-year survival rate of patients with a high serum CEA concentration was significantly lower than that of patients with a normal CEA. There was no difference in the prognostic significance of serum CEA concentration and CEA amount.

Conclusions: We failed to find the association between BMI and CEA, suggesting no or small hemodilution effect of CEA in NSCLC patients. Furthermore, the measurement of the CEA amount could not provide any additional information.

Keywords: Serum CEA; CEA amount; Body mass index; Hemodilution; Non-small cell lung cancer

\section{Introduction}

Carcinoembryonic antigen (CEA) is a glycoprotein involved in

\section{Manuscript accepted for publication April 18, 2017}

aDepartment of Thoracic and Breast Surgery, Faculty of Medicine, University of Miyazaki, Kihara 5200, Kiyotake, Miyazaki 889-1692, Japan

bepartment of Cardiovascular Surgery, Faculty of Medicine, University of Miyazaki, Kihara 5200, Kiyotake, Miyazaki 889-1692, Japan

${ }^{\mathrm{c} C}$ Corresponding Author: Masaki Tomita, Department of Thoracic and Breast Surgery, Faculty of Medicine, University of Miyazaki, Kihara 5200, Kiyotake, Miyazaki 889-1692, Japan. Email: mtomita@med.miyazaki-u.ac.jp

doi: https://doi.org/10.14740/wjon1026w cell adhesion, is used as a tumor marker in a variety of tumors, and plays an important role in the prognosis of non-small cell lung cancer (NSCLC) patients [1-3].

Previous investigations reported that the serum concentration of serum prostate-specific antigen (PSA) level in obese patients with prostate cancer is lower compared with that in non-obese subjects [4-6]. These investigations showed that a lower serum PSA level was associated with increasing body mass index (BMI) [4-6]. The reason for this phenomenon has been considered as a hemodilution effect due to the larger vascular volume of obese patients [4-6]. On the other hand, others found no significant association between BMI and PSA [7, 8]. With regard to CEA, the relation between serum CEA concentration and obesity was also examined in colorectal cancer patients $[9,10]$. However, the number of available studies investigating the association between BMI and serum CEA concentration is limited [9-12]. Despite the abundant literature on serum CEA concentration in NSCLC [1-3], there are no previous investigations on the hemodilution effect on serum CEA concentration in obese NSCLC patients. Therefore, in the present study, we investigated the association between BMI and serum CEA concentration in resected NSCLC patients.

\section{Patients and Methods}

This retrospective study had institutional review board approval, and the need to obtain patient consent was waived. Consecutive NSCLC patients who examined preoperative serum CEA concentration and underwent surgery from 2008 to 2013 in our hospital were enrolled into the present retrospective study. The collected records of 381 consecutive NSCLC patients were reviewed retrospectively.

The preoperative serum CEA concentration $(\mathrm{ng} / \mathrm{mL})$ was measured by enzyme immunoassay in a single laboratory at our hospital. The preoperative BMI was calculated as weight in kilograms divided by height in meters squared. According to the previous investigation [10], the following category was used: low BMI group (BMI $<18.5 \mathrm{~kg} / \mathrm{m}^{2}$ ), normal BMI group (BMI $=18.5-24.0 \mathrm{~kg} / \mathrm{m}^{2}$ ) and high BMI group (BMI $>24.0 \mathrm{~kg} /$ $\mathrm{m}^{2}$ ). Two plasma volume (PV) equations were applied as follows: 1) body surface area (BSA)-based equation: the estimated body surface area (BSA) $\left(\mathrm{m}^{2}\right)$ was calculated as follows: (body weight $)^{0.425} \times(\text { height })^{0.72} \times 0.2025$ [13]. The BSA-based PV $(\mathrm{L})$ was calculated as BSA $\times 1.670$ [14]. 2) Hematocrit $(\mathrm{HCT})-$ based PV $(\mathrm{L})$ was calculated as $0.07 \times$ weight $(\mathrm{kg}) \times(1-\mathrm{HCT})$ [15]. The CEA amount $(\mu \mathrm{g})$, representing the total amount of CEA within the circulation, was calculated as serum CEA 
Table 1. Clinical Characteristics of Patients Based on BMI

\begin{tabular}{|c|c|c|c|c|c|}
\hline & Low BMI & Normal BMI & High BMI & Total & P value \\
\hline \multicolumn{6}{|l|}{ Age } \\
\hline$\leq 65$ & 19 & 68 & 37 & 124 & 0.070 \\
\hline$>65$ & 40 & 149 & 68 & 257 & \\
\hline \multicolumn{6}{|l|}{ Gender } \\
\hline Male & 32 & 120 & 44 & 196 & 0.668 \\
\hline Female & 27 & 97 & 61 & 185 & \\
\hline \multicolumn{6}{|l|}{ Smoking status } \\
\hline Never & 26 & 94 & 51 & 171 & 0.577 \\
\hline Current/former & 33 & 123 & 54 & 210 & \\
\hline \multicolumn{6}{|l|}{ Histology } \\
\hline Adenocarcinoma & 42 & 161 & 91 & 294 & 0.016 \\
\hline Others & 17 & 56 & 14 & 87 & \\
\hline \multicolumn{6}{|l|}{ pStage } \\
\hline I & 42 & 169 & 84 & 295 & 0.433 \\
\hline II-III & 17 & 48 & 21 & 86 & \\
\hline \multicolumn{6}{|l|}{ pT status } \\
\hline pT1 & 33 & 153 & 71 & 257 & 0.114 \\
\hline pT2-3 & 26 & 64 & 34 & 124 & \\
\hline \multicolumn{6}{|l|}{$\mathrm{pN}$ status } \\
\hline $\mathrm{pNO}$ & 51 & 182 & 92 & 325 & 0.645 \\
\hline $\mathrm{pN} 1-2$ & 8 & 35 & 13 & 56 & \\
\hline \multicolumn{6}{|l|}{ CEA } \\
\hline Normal & 39 & 160 & 80 & 279 & 0.375 \\
\hline High & 20 & 57 & 25 & 102 & \\
\hline
\end{tabular}

CEA: carcinoembryonic antigen; BMI: body mass index.

concentration $\times$ estimated PV. Both CEA amount estimated by BSA and HCT were calculated. To determine the cut-off value of CEA amount, receiver operating characteristics (ROC) curve of CEA amount was analyzed, and cancer death was predicted by comparing the area under the curve. We decided the best cutoff value for BSA-based CEA amount was 12.09 (sensitivity: $78.65 \%$; specificity: $50.0 \%$; area under the ROC curve: 0.679 ) and HCT-based CEA amount was 10.52 (sensitivity: 77.58\%; specificity: $52.0 \%$; area under the ROC curve: 0.669$)$.

Cumulative survival curves after surgery were calculated using the Kaplan-Meier method and differences were evaluated using the log-rank test. We used Wilcoxon rank-sum tests to assess associations between BMI and PV, serum CEA concentration and CEA amount, with P-values computed using the normal approximation. All statistical analyses were performed using JMP (SAS Institute Inc., Cary, NC, USA).

\section{Results}

The clinicopathological factors of patients based on BMI group were shown in Table 1. Among these NSCLC patients,
$15.5 \%$ were categorized as low BMI, $57 \%$ as normal BMI, and $27.5 \%$ as high BMI. Age, gender, smoking status, pStage, pT status, $\mathrm{pN}$ status, and serum CEA concentration were not different between BMI groups. However, high BMI group had a statistically significant association with increasing adenocarcinoma histology $(\mathrm{P}=0.016)$.

As shown in Table 2, the BSA-based PV was significantly increased with higher BMI $(\mathrm{P}<0.001)$. Similarly, the HCTbased PV was also significantly associated with higher BMI $(\mathrm{P}<0.001)$. However, there was no association between serum CEA concentration and BMI $(\mathrm{P}=0.171)$. We could not find even the trend towards an association between increasing BMI and decreasing CEA.

The BSA-based CEA amount had no association with BMI $(\mathrm{P}=0.842)$. The HCT-based CEA amount was not also significantly associated with increasing BMI $(\mathrm{P}=0.598)$.

The survival of patients with preoperative high serum CEA concentration was significantly poorer than those with preoperative normal serum CEA ( $\mathrm{P}<0.001$; Fig. 1a). Similarly, NSCLC patients with high CEA amount had a significantly worse survival than those with low CEA amount $(\mathrm{P}<0.001$; Fig. 1b, c). As shown in Figure 1, the result of BSA-based CEA 
Table 2. Plasma Volume, Serum CEA Concentration and CEA Amount Based on BMI

\begin{tabular}{lllll}
\hline & Low BMI & Normal BMI & High BMI & P value \\
\hline BSA-based PV & $2.3 \pm 0.2$ & $2.5 \pm 0.2$ & $2.7 \pm 0.3$ & $<0.001$ \\
HCT-based PV & $1.8 \pm 0.3$ & $2.3 \pm 0.3$ & $2.7 \pm 0.4$ & $<0.001$ \\
Serum CEA concentration & $28.1 \pm 3.7$ & $12.2 \pm 0.8$ & $15.5 \pm 1.5$ & 0.171 \\
BSA-based CEA amount & $25.5 \pm 64.8$ & $15.1 \pm 31.6$ & $15.4 \pm 41.8$ & 0.842 \\
HCT-based CEA amount & $20.1 \pm 52.5$ & $13.6 \pm 29.2$ & $15.1 \pm 41.1$ & 0.598 \\
\hline
\end{tabular}

All values are expressed as mean \pm standard deviation. BMl: body mass index; BSA: body surface area; HCT: hematocrit; PV: plasma volume; CEA: carcinoembryonic antigen.

amount was consistent with that of HCT-based CEA amount. Both CEA amount could not provide any useful information in addition to serum CEA concentration.

\section{Discussion}

We demonstrated that high BMI patients had higher PV, in agreement with the results of previous investigations [4-6]. It was also previously demonstrated that the serum PSA concentration in obese individuals was lower compared with normalweight individuals [4-6]. The hemodilution may therefore be a most considerable reason for the lower serum PSA concentrations among obese men with prostate cancer [4-6]. Compared with theses previous investigations, we failed to find no association between serum CEA concentration and BMI in NSCLC patients. In agreement with our results, some investigations also found no significant association between BMI and PSA $[7,8]$. The reason for this discrepancy is unknown. Some possible reasons for this might be as follows. First, the present study represents a retrospective surgical cohort from a single institution. The characteristics of resected NSCLC patients may not reflect the all staged NSCLC patients and/or general population. Second, the present study is the first investigation for NSCLC. There are no previous investigations that showed the relationship between BMI and serum CEA concentration in NSCLC. Third, in the present study, the number of extremely obese Japanese patients is small. The patients with BMI $>27.5$ and $>30$ were only $5.0 \%(19 / 381)$ and $1.3 \%(5 / 381)$ of our study population, respectively. Yoshiike et al [16] reported that the standardized prevalence of obesity (BMI $\geq 30.0)$ in Japa- nese adults was quite low compared with the data in western populations. Therefore, because of small number of obese patients, there is a possibility that we might fail to find a hemodilution of CEA in Japanese NSCLC patients.

We found a statistical significant association between high BMI and higher PV, but not found the association between high BMI and serum CEA concentration. Therefore, it is easy to understand that increasing BMI is associated with increasing CEA amount. However, our result showed that there was no association between BMI and CEA amount in NSCLC patients. Therefore, the power of the obesity-related CEA hemodilution in NSCLC patients might be weak, and the hemodilution effect in the obese NSCLC patients might be clinically negligible.

Previous investigations showed the prognostic significance of serum CEA concentration in NSCLC [1-3]. If the power of the hemodilution effect in the obese NSCLC patients is strong, the prognostic significance of CEA amount might be more useful than that of serum CEA concentration. However, we could not find any differences between the survival curve based on serum CEA concentration and that based on CEA amount. Taken together, there might be no need to adjust the preoperative serum CEA concentration in relation to the BMI when used to predict the prognostic assessment in NSCLC patients.

The main limitations of our study are that all patients were surgery patients and the number of patients was small. Therefore, a large cohort study will be required to confirm our results.

\section{Conclusions}

In conclusions, we failed to find the association between BMI
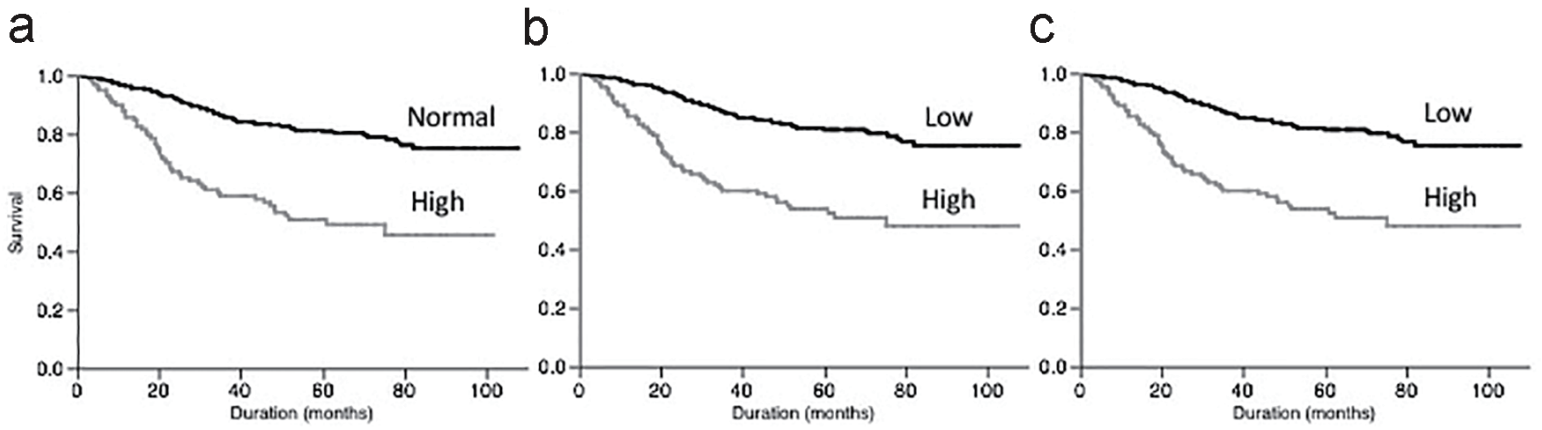

Figure 1. Survival of patients according to serum CEA concentration (a), BSA-based CEA amount (b) and HCT-based CEA amount (c). 
and CEA, suggesting the hemodilution effect in the obese NSCLC patients might be clinically negligible. Furthermore, the measurement of the CEA amount could not provide any additional information. Therefore, we believe that there is no need to adjust the preoperative serum CEA concentration in relation to the BMI in NSCLC patients.

\section{Conflicts of Interest}

The authors have declared that no conflicts of interest exist.

\section{References}

1. Okada M, Nishio W, Sakamoto T, Uchino K, Yuki T, Nakagawa A, Tsubota N. Prognostic significance of perioperative serum carcinoembryonic antigen in non-small cell lung cancer: analysis of 1,000 consecutive resections for clinical stage I disease. Ann Thorac Surg. 2004;78(1):216221.

2. Sawabata N, Maeda H, Yokota S, Takeda S, Koma M, Tokunaga T, Ito M. Postoperative serum carcinoembryonic antigen levels in patients with pathologic stage IA nonsmall cell lung carcinoma: subnormal levels as an indicator of favorable prognosis. Cancer. 2004;101(4):803809.

3. Tomita M, Matsuzaki Y, Edagawa M, Shimizu T, Hara M, Onitsuka T. Prognostic significance of preoperative serum carcinoembryonic antigen level in lung adenocarcinoma but not squamous cell carcinoma. Ann Thorac Cardiovasc Surg. 2004;10(2):76-80.

4. Banez LL, Hamilton RJ, Partin AW, Vollmer RT, Sun L, Rodriguez C, Wang Y, et al. Obesity-related plasma hemodilution and PSA concentration among men with prostate cancer. JAMA. 2007;298(19):2275-2280.

5. Werny DM, Thompson T, Saraiya M, Freedman D, Kottiri BJ, German RR, Wener M. Obesity is negatively associated with prostate-specific antigen in U.S. men, 2001-2004. Cancer Epidemiol Biomarkers Prev. 2007;16(1):70-76.

6. Chang IH, Ahn SH, Han JH, Kim TH, Kim YS, Myung
SC. The clinical significance in healthy men of the association between obesity related plasma hemodilution and tumor marker concentration. J Urol. 2009;181(2):567572.

7. Freedland SJ, Platz EA, Presti JC, Jr., Aronson WJ, Amling CL, Kane CJ, Terris MK. Obesity, serum prostate specific antigen and prostate size: implications for prostate cancer detection. J Urol. 2006;175(2):500-504.

8. Mitchell CR, Umbreit EC, Rangel LJ, Bergstralh EJ, Karnes RJ. Does body mass index "dilute" the predictive property of prostate-specific antigen for tumor volume at radical prostatectomy? Urology. 2011;78(4):868-872.

9. Park JS, Choi GS, Jang YS, Jun SH, Kang H. Influence of obesity on the serum carcinoembryonic antigen value in patients with colorectal cancer. Cancer Epidemiol Biomarkers Prev. 2010;19(10):2461-2468.

10. Chen W, Liu Q, Tan SY, Jiang YH. Association between carcinoembryonic antigen, carbohydrate antigen 19-9 and body mass index in colorectal cancer patients. Mol Clin Oncol. 2013;1(5):879-886.

11. Li F, Shen Z, Lu Y, Wang L, Song W. How much does body mass index affect serum carcinoembryonic antigen concentration? Cancer Epidemiol Biomarkers Prev. 2014;23(3):555-560.

12. Park M, Chang IH, Kang H, Han SS. Effect of obesityrelated plasma hemodilution on serum tumor marker concentration in women. J Obstet Gynaecol Res. 2015;41(5):784-789.

13. Du Bois D, Du Bois EF. A formula to estimate the approximate surface area if height and weight be known. 1916. Nutrition. 1989;5(5):303-311; discussion 312-303.

14. Boer P. Estimated lean body mass as an index for normalization of body fluid volumes in humans. Am J Physiol. 1984;247(4 Pt 2):F632-636.

15. Kaplan AA. A simple and accurate method for prescribing plasma exchange. ASAIO Trans. 1990;36(3):M597599.

16. Yoshiike N, Matsumura Y, Zaman MM, Yamaguchi M. Descriptive epidemiology of body mass index in Japanese adults in a representative sample from the National Nutrition Survey 1990-1994. Int J Obes Relat Metab Disord. 1998;22(7):684-687. 